\title{
Structural model of Cowlesite by fast electron diffraction tomography
}

Mauro Gemmi ${ }^{1}$, Enrico Mugnaioli ${ }^{1}$, Jeremy David ${ }^{1}$, Giuseppe Cruciani ${ }^{2}$, Marco Merlini ${ }^{3}$

${ }^{1}$ Istituto Italiano Di Tecnologia, Pisa, Italy, ${ }^{2}$ Dipartimento di Fisica e Scienze della Terra, Università di Ferrara, Ferrara, Italy, ${ }^{3}$ Dipartimento di Scienze della Terra "A. Desio" Università degli Studi di Milano, Milano, Italy E-mail: mauro.gemmi@iit.it

Cowlesite is a natural Ca-rich zeolite with ideal chemical formula Ca6Al12Si18 060 . 36H2O. The small size of the lath-like crystals and their disordered intergrowth nature hampered proper single crystal X-ray data studies, while all the attempts to solve the structure with powder X-ray diffraction data (proposed orthorhombic $23.3 \times 25.0 \times 30.6 \AA$ unit cell [1]) failed. The. We studied a powder sample of Cowlesite using the recently developed fast electron diffraction tomography technique [2], in which a sequence of electron diffraction patterns is collected while the crystal is continuously rotated around the sample holder axis. We used this fast procedure since Cowlesite is beam sensitive and long illumination induces amorphization of the sample. The patterns are collected in precession mode and the explored angular range is limited by the stability of the TEM goniometer, which does not guarantee that the investigated crystal stays inside the beam for the whole available rotational range. In order to have an acceptable reciprocal space coverage we stitched together four consecutive data collection from the same crystal. The unit cell we measured is still orthorhombic, $23.0 \times 24.4 \times 25.4 \AA$, but the third parameter is significantly shortened. We observe strong streaking along (00I), the direction of shrinking, while in hk0 planes the reflections are sharply defined, suggesting the OD nature of Cowlesite. The (family) structure of the framework can be solved in space group Ccme and it is formed by 2D blocks stacked along [00I] and connected by just four tetrahedra, which leaves a huge cavity in between. Half of the expected $\mathrm{Ca}$ atoms and several water molecules are missing. Large 10 and 8 ring channels run parallel to $c$ and [110] directions respectively. The shortened unit cell we found does not index the Cowlesite Xray powder pattern, therefore we think that the structure partially dehydrates in the high vacuum of the microscope, and the model we obtain describes the dehydrated Cowlesite. We can guess a possible model for the hydrated Cowlesite, which requires a separation of the framework blocks and an interlayer rich of water and Ca atoms. In such a case Cowlesite would be the only example of a natural zeolite possessing a layered structure similar to synthetic 2D zeolites [3].

[1] Vezzalini, G. et al. (1992) Min. Mag. 56, 575-579.

[2] Gemmi, M. et al. (2015) J. Appl.Cryst. 48, 718-727.

[3] Zanardi, S. et al. (2004) Angew. Chem. Int. Ed. Engl. 43, 4933-7.

Keywords: Electron diffraction tomography, Zeolites, Transmission electron microscopy 